\title{
Comparación entre dos escalas de medida subjetiva: Thurstone y Anderson
}

\section{Angela Conchillo Jiménez*}

Universidad Complutense

\section{INTRODUCCION}

Uno de los grandes empeños de la Psicología ha sido obtener escalas de medida para variables no físicas como, por ejemplo, la inteligencia, actitudes, sensación, etc. La psicofísica ha tenido como principal objetivo la obtención de una función, $\mathrm{S}=\mathrm{f}(\mathrm{E})$, que atribuyese a cada valor del estímulo físico un valor correspondiente a la sensación producida por el mismo. Pero la obtención de escalas de medida para variables subjetivas no ha estado limitada únicamente al ámbito de la Psicofísica. Esto es, la Psicología en su empeño por fundamentar la medición de variables psicológicas no se ha limitado exclusivamente a aquellas variables que, como la sensación, tienen un correlato físico conocido, sino que ha ido más allá buscando escalas de medida para estímulos subjetivos, es decir, para estímulos sin este correlato físico.

Dos teorías que se han ocupado de obtener escalas de medida para estímulos subjetivos han sido la Ley del Juicio Comparativo de Thurstone (1927 a, b, c; 1928; 1959) y la Teoría de la Integración de la Información de Anderson (1974 a, b, c; 1981; 1982). Ambas teorías, fundamentándose en supuestos distintos, pero no incompatibles, pretenden ofrecer una escala de intervalos para estímulos subjetivos.

Mientras que para Thurstone la obtención de esta escala es el modelo mismo del Juicio Comparativo, para Anderson el objetivo fundamental, no es tanto la obtención de la escala, sino la búsqueda de una función, la Ley Psicológica, que formalice cómo se integra la información contenida en diversos estímulos a fin de proporcionar una determinada respuesta. Pero la Función de Integración o Ley Psicológica da lugar a una escala de medida para los estímulos que han sido integrados.

Aunque Anderson reconoce que el trabajo de Thurstone ha sido de fundamental importancia y está en la base de la Teoría de la Integración de la Información, sin embargo, no existen estudios que hayan intentado relacionar estas dos teorías. El objetivo de esta investigación va en esta línea: Nos encontramos con dos teorías, la Ley del Juicio Comparativo y la Teoría de la Integración de la Información. Ambas proponen, desde supuestos distintos, pero no incompatibles, una medida en escala de intervalos para estímulos subjetivos. Si ello es así, utilizando los mismos estímulos y los mismos sujetos, cabría esperar la existencia de una transfor- 
mación admisible para la escala de intervalos que nos permitiese pasar de la escala de medida proporcionada por un modelo a la proporcionada por el otro. Es decir, debe existir una función lineal con pendiente positiva que relacione ambos conjuntos numéricos.

Pero antes de describir la metodología utilizada para alcanzar este objetivo, vamos a exponer una síntesis de los dos modelos que nos ocupan, a fin de que el lector pueda comprender tanto la metodología como el objetivo de esta investigación.

\section{LA LEY DEL JUICIO COMPARATIVO DE THURSTONE}

Thurstone (1927 a,b) pensó que la Psicofísica tenía un campo muy reducido de aplicación, pues se limitaba a la búsqueda de una función que relacionase la sensación con el estímulo físico correspondiente. ¿No sería posible atribuir valores en el continuo subjetivo a estímulos más cualitativos, es decir, a estímulos que no tienen un correlato físico? Cuando el estímulo no tiene correlato físico conocido surge un nuevo problema, a saber, no tenemos unos valores obtenidos mediante medición física con los cuales podamos contrastar nuestra medición psicológica. Así, si un sujeto jụzga que un estímulo $A$ es mejor que un estímulo $B$ el 75 por 100 de las veces en que ambos estímulos son comparados y que el estímulo $B$ es mejor que un estímulo $C$ el 60 por 100 de las veces, podemos establecer una relación de orden, " $A$ es mejor que $B$ y $B$ es mejor que $C$ », pero no sabemos cuál es la distancia entre A y $B$ y entre $B$ y $C$. Es decir, esta relación nos proporciona una escala ordinal pero no una escala de intervalos. Anteriormente Thorndike había solucionado el problema asumiendo que la diferencia entre las distancias es proporcional a la diferencia entre las desviaciones típicas correspondientes a las proporciones. Thurstone da un paso más y en este sentido propone un modelo matemático para relacionar los valores numéricos atribuidos a un conjunto de estímulos con las proporciones observadas.

Según Thurstone, la Ley del Juicio Comparativo es básica a todo trabajo experimental que asuma la Ley de Weber y la de Fechner, pero además tiene otro ámbito de aplicación, el de la construcción de escalas psicológicas y educativas que impliquen juicios comparativos. El mismo llega a decir (1959, pág. 15) que, de todo lo que ha escrito, su mejor aportación a la Psicología fue su artículo «Psychological Analysis» (1927) en el que trata la Ley del Juicio Comparativo y sus implicaciones. Pero hay que decir que esta afirmación se produce treinta y dos años después y que en ese período Thrustone no dedicó sus esfuerzos al perfeccionamiento, difusión y aplicación experimental de su ley.

Anderson (1981) dice, refiriéndose a la Ley del Juicio Comparativo, que, aun cuando pudo aplicarse para resolver la polémica establecida en torno a la igualdad o no de las j.n.d. y, por tanto, en la determinación de la Ley Psicofísica, no tuvo mucho impacto en este campo de la Psicofísica. Una de las razones, según Anderson, de este poco impacto fue que el propio Thurstone no dedicó demasiado tiempo a la aplicación de su Ley, dedicándose durante más de dos décadas al estudio factorial de la inteligencia.

Pero veamos cuál es el modelo básico que subyace al trabajo de Thurstone.

Presentamos una serie de estímulos a los cuales el sujeto puede responder diferencialmente con respecto a algún atributo de ellos. Nuestra tarea consiste en localizar cada estímulo sobre un continuo psicológico de 
tal modo que explique las respuestas dadas por el observador. El continuo psicológico puede ser considerado como un continuo subjetivo o de magnitudes psicológicas. Según Thurstone, cuando presentamos un estímulo a un sujeto aparece un proceso discriminativo y este proceso tiente un valor en el continuo psicológico. Un proceso discriminativo es aquel por el cual el organismo identifica, distingue o reacciona ante los estímulos.

Debido a fluctuaciones momentáneas del organismo, un estímulo cualquiera no excita siempre el mismo proceso discriminativo, es decir, el sujeto no le atribuirá siempre el mismo valor sobre el continuo subjetivo, sino que en algunas ocasiones atribuirá valores algo mayores o menores. Como resultado de esta fluctuación, en lugar de un único proceso discriminativo asociado con un estímulo, tendremos una distribución de procesos discriminativos que están asociados con el estímulo en cuestión. Thurstone supone que esa distribución es normal.

De todos los procesos discriminativos asociados con un mismo estímulo habrá alguno que aparezca mayor número de veces, a éste se le llama proceso discriminativo modal. Como se supone una distribución normal, el proceso discriminativo modal es, asimismo, la media y la mediana de todos los procesos discriminativos asociados con ese estímulo.

La desviación típica de la distribución de procesos discriminativos asociados con el estímulo se llama dispersión discriminativa del estímulo y no tiene por qué ser igual para los distintos estímulos.

El valor de escala que se asigna a cada estímulo es la media de todos los procesos discriminativos asociados a ese estímulo y, como ya hemos señalado, es igual al proceso discriminativo modal.

Establecidos estes conceptos básicos del modelo de Thurstone veamos en qué consiste y cómo se deduce la Ley del Juicio Comparativo.

Propiamente, la Ley del Juicio Comparativo es una ecuación que relaciona la proporción de veces que un estímulo, $\mathrm{E}_{\mathrm{k}}$, es juzgado ser mayor (más preferido, más brillante, mejor, etc.) que otro estímulo, $\mathrm{E}_{\mathrm{j}}$, con los procesos discriminativos modales de estos dos estímulos.

Supongamos dos estímulos, $\mathbf{E}_{\mathbf{j}}$ y $\mathrm{E}_{\mathbf{k}}$. Cada uno de estos estímulos tendrá asociada una distribución de procesos discriminativos que, como ya se ha señalado, es normal.

Sean $S_{k}$ y $S_{j}$ los procesos discriminativos modales correspondientes a la distribución asociada a $\mathrm{E}_{\mathrm{k}}$ y $\mathrm{E}_{\mathrm{j}}$, respectivamente, y sean $\delta_{\mathrm{k}}$ y $\delta_{\mathrm{j}}$ las correspondientes dispersiones discriminativas.

Supongamos que presentemos simultáneamente estos dos estímulos, $E_{j}$ y $E_{k}$, a un sujeto en repetidas ocasiones. En cada ocasión tendremos dos procesos discriminativos $y$, consiguientemente, una diferencia entre ambos. Esta diferencia se llama diferencia discriminativa y tiene una distribución normal ya que son normales las distribuciones de los procesos discriminativos asociados a cada estímulo. La media de la distribución de diferencias es la diferencia de las medias de las distribuciones correspondientes a cada estímulo, es decir,

$$
\overline{\mathrm{S}}_{\mathrm{k}-\mathrm{i}}=\overline{\mathrm{S}}_{\mathrm{k}}-\overline{\mathrm{S}}_{\mathrm{i}}
$$

La desviación típica de la distribución de diferencias es

$$
\delta_{\mathrm{kj}}=\left(\delta_{\mathrm{j}}^{2}+\delta_{\mathrm{k}}^{2}-\mathrm{r}_{\mathrm{jk}} \delta_{\mathrm{k}} \delta_{\mathrm{j}}\right)^{1 / 2}
$$

donde

$\mathrm{r}_{\mathrm{jk}}$ es la correlación entre los valores de los procesos discriminativos asociados a los estímulos $\mathrm{E}_{\mathrm{j}} \mathrm{y} \mathrm{E}_{\mathrm{k}}$. 
Considerando la media de las diferencias, $\mathrm{S}_{\mathrm{k}-\mathrm{j}}$, puede ocurrir uno de los tres casos siguientes:

a) $\bar{S}_{k-j}=0$ si y sólo si $\bar{S}_{k}=\bar{S}_{j}$

b) $\bar{S}_{k-j}>0$ si y sólo si $\bar{S}_{k}>\bar{S}_{j}$

c) $\overline{\mathrm{S}}_{\mathrm{k}-\mathrm{j}}<0$ si y sólo si $\overline{\mathrm{S}}_{\mathrm{k}}<\overline{\mathrm{S}}_{\mathrm{j}}$

Consideremos la diferencia $\bar{S}_{\mathrm{k}-\mathrm{j}}$ expresada en unidades típicas, $z_{\mathrm{kj}}$. Podemos averiguar el valor $z_{k j}$ a partir de la proporción de veces que el sujeto juzga que el estímulo $E_{k}$ es mayor que el estímulo $E_{j}$.

Anteriormente. hemos señalado que los dos estímulos, $E_{k}$ y $E_{j}$, se presentan al mismo sujeto en un gran número de ocasiones y vamos a considerar como equivalente presentar los dos estímulos en una sola ocasión a un gran número de sujetos, añadiendo, que, por razones metodológicas, no se permite juzgar los dos estímulos como iguales en aquella dimenisión en que se están comparāndo.

Sea

$\mathrm{N}=\mathrm{Número}$ de ocasiones que se presentan simultáneamente $\mathrm{E}_{\mathrm{k}}$ y $\mathrm{E}_{\mathrm{j}}$. Es decir, número de comparaciones realizadas con estos dos estímulos.

$\mathrm{f}_{\mathrm{kj}}=$ Númeró de veces que el estímulo $\mathrm{E}_{\mathrm{k}}$ es juzgado ser mayor el estímulo $\mathrm{E}_{\mathrm{j}}$.

$P_{k j}=f_{k j} / N$ es la proporción de veces que el estímulo $E_{k}$ es juzgado ser mayor que el estímulo $\mathrm{E}_{\mathrm{j}}$.

Reconsideremos los tres casos anteriores:

a) $\overline{\mathrm{S}}_{\mathrm{k}-\mathrm{i}}=0$ si y sólo si $\overline{\mathrm{S}}_{\mathrm{k}}=\overline{\mathrm{S}}_{\mathrm{j}}$

Si $\bar{S}_{k}=\widehat{S}_{j}$ es que los dos estímulos, $E_{k}$ y $E_{j}$, tienen el mismo valor subjetivo para el sujeto, en cuyo caso debe esperarse que el sujeto juzgue que el estímulo $E_{k}$ es mayor que el estímulo $E_{j}$ el 50 por 100 de las veces $y$ que $E_{j}$ es mayor que $E_{k}$ otro 50 por 100 de las veces, dado que no se permite juzgar que un estímulo es igual a otro. En esta situación, $\mathrm{p}_{\mathrm{kj}}=0.5 \mathrm{y}$ la puntuación típica que le corresponde, supuesta la normalidad de la distribución, es $z_{\mathrm{kj}}=0$.

b) $\overline{\mathrm{S}}_{k-\mathrm{j}}>0$ si y sólo si $\overline{\mathrm{S}}_{\mathrm{k}}>\overline{\mathrm{S}}_{\mathrm{j}}$

Si $\bar{S}_{k}>\bar{S}_{j}$ entonces cabe esperar que el sujeto juzgue en más de la mitad de los casos que el estímulo $\mathrm{E}_{\mathrm{k}}$ es mayor que el estímulo $\mathrm{E}_{\mathrm{j}}$, en cuyo caso tendremos que $\mathrm{p}_{\mathrm{kj}}>0.5$. Igualmente podemos obtener, supuesta la normalidad de la distribución, cual es el valor típico, $\mathrm{z}_{\mathrm{k} j}$, que le corresponde.

c) $\bar{S}_{k-j}<0$ si y sólo si $\bar{S}_{k}<\bar{S}_{j}$

Si $\bar{S}_{k}<\bar{S}_{j}$ entonces debemos esperar que el sujeto juzgue en menos de la mitad de los casos que el estímulo $E_{k}$ es mayor que el estímulo $E_{j}$, luego $\mathrm{P}_{\mathrm{kj}}<0.5$. Como en los casos anteriores podemos obtener mediante la distribución normal la correspondiente puntuación típica, $z_{\mathbf{k j}}$.

Ahora bien, sabemos que $\bar{S}_{k-j}=\bar{S}_{k}-\bar{S}_{j}$ y $\bar{S}_{k-j}=z_{k j} \delta_{k j}$

luego, $\bar{S}_{\mathrm{k}}-\overline{\mathrm{S}}_{\mathrm{j}}=z_{\mathrm{kj}}\left(\delta_{\mathrm{j}}^{2}+\delta_{\mathrm{k}}^{2}-2 \mathrm{r}_{\mathrm{kj}} \delta_{\mathrm{j}} \delta_{\mathrm{k}}\right)^{1 / 2}$

Esta ecuación es la Ley del Juicio Comparativo.

Ahora bien, la Ley del Juicio Comparativo, así formulada, es inoperativa, no puede ser aplicada. Pensemos que con $n$ estímulos tendremos $\mathrm{C}_{\mathrm{n}, 2}=\mathrm{n}(\mathrm{n}-1) / 2$ pares de estímulos $\mathrm{y}$, por tanto, $\mathrm{C}_{\mathrm{n}, 2}$ ecuaciones distintas. Los únicos valores conocidos son las $\mathrm{z}_{\mathrm{kj}}$ correspondientes en cada ecuación. En cambio los valores desconocidos son:

n procesos discriminativos

$\mathrm{n}$ dispersiones discriminativas $\mathrm{y}$

$\mathrm{C}_{\mathrm{n}, 2}$ correlaciones.

Por tanto, tenemos más incógnitas que ecuaciones. Ello nos lleva a imponer restricciones al modelo. Thurstone presenta cinco casos o formulaciones de la Ley del Juicio Comparativo, según sea el tipo de restricción 
que se imponga. De estos cinco casos nosotros nos vamos a fijar en el Caso V que es el más simplificado y, por ello, el más operativo.

$\mathrm{El}$ Caso $\mathrm{V}$ impone las dos restricciones siguientes:

$$
\begin{array}{ll}
\delta_{\mathrm{j}}=\delta_{\mathrm{k}}= & , \forall_{\mathrm{j}, \mathrm{k}} \\
\mathbf{r}_{\mathrm{jk}}=\mathrm{r} & , \forall_{\mathrm{j}, \mathrm{k}}
\end{array}
$$

Bajo estas dos restricciones la Ley del Juicio Comparativo es:

$$
\begin{aligned}
\bar{S}_{\mathrm{k}}-\bar{S}_{\mathrm{j}} & =z_{\mathrm{kj}}\left(2 \delta^{2}-2 r \delta^{2}\right)^{1 / 2} \\
& =z_{\mathrm{kj}}\left(2 \delta^{2}(1-\mathrm{r})\right)^{1 / 2}
\end{aligned}
$$

Ahora bien, puesto que $\left[2 \delta^{2}(1-r)\right]^{1 / 2}$ es constante para todo par de estímulos podemos considerarlo como una unidad de medida, en cuyo caso la Ley del Juicio Comparativo es

$$
\overline{\mathrm{S}}_{\mathrm{k}}-\overline{\mathrm{S}}_{\mathrm{j}}=\mathrm{z}_{\mathrm{kj}}
$$

Esta ecuación nos permite atribuir un valor numérico a cada estímulo. Hemos de señalar que el modelo no es operativo cuando $\mathrm{p}_{\mathrm{kj}}=1$ ó $\mathrm{p}_{\mathrm{kj}}=0$, ya que no es posible obtener la correspondiente puntuación típica.

Después de esta exposición de la Ley del Juicio Comparativo conviene tener presente que la finalidad de Thurstone era obtener una escala de intervalos para estímulos subjetivos, y que este objetivo se consigue después de imponer ciertas restricciones al modelo.

Vamos ahora a exponer la Teoría de la Integración de Anderson que, desde supuestos distintos, permite obtener también una escala de intervalos para estímulos subjetivos.

\section{LA TEORIA DE LA INTEGRACION DE LA INFORMACION DE ANDERSON}

La Teoría de la Integración de la Información ha sido formulada por Anderson a partir de un conjunto de investigaciones llevadas a cabo desde el comienzo de la década del sesenta hasta nuestros días.

Los fundamentos teóricos, la metodología y la aplicación experimental de esta teoría han sido sintetizados y publicados recientemente (Anderson, 1981, 1982).

Como ya se hà señalado, en esta teoría se recoge la tradición de Thurstone en el sentido de que permite obtener una escala de intervalos, no sólo para la sensación proporcionada por estímulos físicos, sino también para la medición de estímulos subjetivos como los utilizados por Thurstone. Aunque conviene señalar que el objetivo primero de la Teoría de la Integración de la Información no es la obtención de esta escala de medida, sino, más bien, ofrecer un modelo que explique cómo se integra la información contenida en diversos estímulos a fin de producir una respuesta determinada. A partir de de esta matriz de respuestas, como una consecuencia del modelo de integración, se puede obtener una escala de medida para los diversos estímulos integrados. Pero veamos cuáles son los conceptos básicos en los cuales se apoya esta teoría.

\section{Conceptos Básicos}

La Teoría de la Integración de la Información se maneja con tres conceptos básicos, a saber, el concepto de integración, el de valoración y el de álgebra cognitiva.

El concepto de integración hace referencia a que cualquier respuesta depende de la integración proporcionada por un conjunto de estímulos. 
El concepto de valoración hace referencia a que cualquier estímulo tiene una valoración psicológica, es decir, hay un medida interna de ese estímulo. Esta valoración o medida interna existe tanto para los estímulos físicos como para aquellos otros que no tienen un correlato físico conocido.

Por último, el concepto de álgebra cognitiva se refiere a que la integración de estos estímulos a menudo obedece reglas algebraicas simples. Para llegar a una determinada respuesta el sujeto puede sumar, multiplicar, dividir, etc, los valores que internamente ha otorgado a los distintos estímulos. A estas reglas, que no necesariamente verifican todas las propiedades del álgebra matemática, es a lo que se llama álgebra cognitiva.

Los tres conceptos mencionados se integran en el siguiente diagrama de medida:

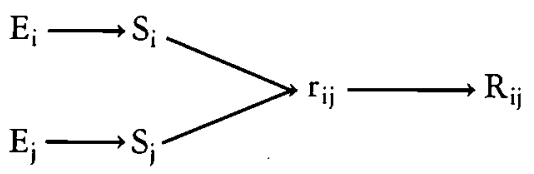

La explicación de este diagrama de medida es como sigue:

En un primer paso los estímulos, físicos o no, son valorados internamente. Como ya sabemos, la función que relaciona la medida física del estímulo con su medida psicológica es conocida con el nombre de Ley Psicofísica.

En un segundo paso, los valores internos atribuidos a los estímulos son integrados mediante alguna regla algebraica dando lugar a una respuesta interna. A la función que relaciona los valores subjetivos de los estímulos con esta respuesta interna se la llama Ley Psicológica.

Por último, en un tercer paso, la respuesta interna obtenida mediante la integración de los valores subjetivos de los estímulos da lugar a una respuesta externa. La función que relaciona la respuesta interna con la externa es la Ley Psicomotora.

La teoría que nos ocupa tiene como objetivo fundamental la obtención de la Ley Psicológica, es decir, la obtención de la función mediante la cual los estímulos son combinados e integrados para producir una respuesta. Según sea esta función nos encontraremos con uno u otro modelo de integración de la información. Así, por ejemplo, si los valores subjetivos de los estímulos son sumados estamos ante un modelo aditivo de integración, si, por el contrario, se multiplican el modelo será multiplicativo, etc.

Aunque el objetivo fundamental de la teoría de Anderson es la búsqueda de la función de integración, esta función permite obtener una escala de medida, tanto para los estímulos como para la respuesta interna. Así, pues, sintetizando, los objetivos que se alcanzan en la teoría que nos ocupa son:

a) Determinación de la Ley Psicológica o función de integración.

b) Obtención de una escala de intervalos en la medición del valor psicológico de los estímulos.

c) Obtención de una esćala de intervalos en la medición del valor psicológico de la respuesta interna.

Anderson utiliza el término «medida funcional» para referirse a las escalas de medida obtenidas, tanto para el estímulo como para la respuesta, puesto que es la forma matemática de la función de integración la que, implícitamente, permite obtener estas escalas de medida.

Los modelos de integración que han sido estudiados por Anderson han sido diversos, pero nosotros vamos a exponer sólo dos de ellos, a saber, el modelo aditivo y el modelo multiplicativo, que son lo más utilizados. 


\section{El Modelo Aditivo}

Consideremos dos continuos estimulares, $\mathrm{A}$ y $\mathrm{B}$.

$A_{1}, A_{2}$ son los diversos valores que toma $A$

$\mathrm{B}_{1}, \mathrm{~B}_{2}$ son los diversos valores que toma $B$.

Supongamos que a un sujeto se le presentan los posibles pares de estímulos, $A_{i} B_{j}$, donde $i: 1,2 \ldots$. y $j: 1,2 \ldots J$., pidiéndole que otorgue una puntuación numérica, $\mathrm{R}_{\mathrm{ij}}$, en una variable que supuestamente está relacionada con los estímulos A y B.

Por ejemplo, A puede ser un conjunto de adjetivos que hagan referencia al carácter de una hipotética persona, y $\mathrm{B}$ puede ser un conjunto de verbos que hagan referencia a acciones de esa hipotética persona.

Supongamos:

A: amistoso, equilibrado, desagradable

B: odiar, criticar, aconsejar, ayudar

A un sujeto se le presentan todos los posibles pares (adjetivo - verbo) para que puntúe la «amabilidad» de una hipotética persona descrita por el par presentado.

Las puntuaciones o respuestas dadas por el sujeto ante cada par $\left(A_{i}\right.$, $B_{j}$ ) pueden ordenarse en una tabla como la que sigue

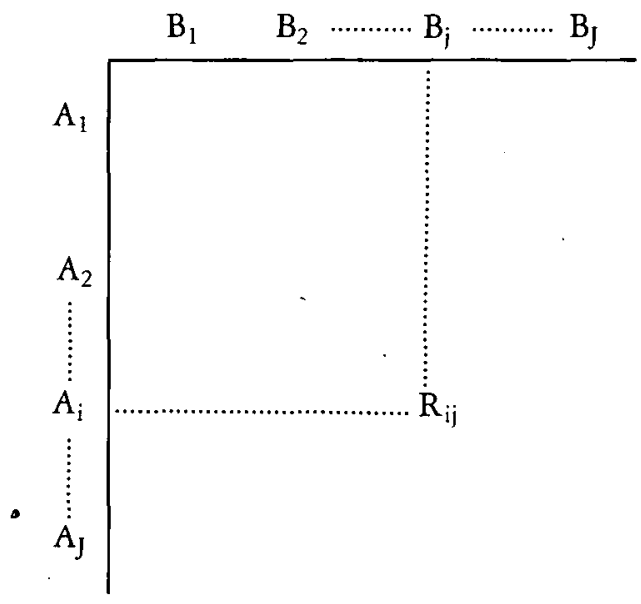

donde $\mathrm{R}_{\mathrm{ij}}$ es la puntuación numérica (respuesta empírica) otorgada por el sujeto ante el par $A_{i}, B_{j}$.

Los supuestos del modelo aditivo son:

a) $r_{i j}=S_{A i}+S_{B J}$, donde,

$r_{i j}$ es la respuesta interna del sujeto ante el par $A_{i}, B_{J}$.

$S_{A i}$ y $S_{B j}$ son los valores subjetivos de los estímulos $A_{i}$ y $B_{j}$, respectivamente.

Este supuesto establece que la función de integración es aditiva, ya que la respuesta interna es la suma de los valores subjetivos de los estímulos.

b) $R_{i j}=C_{0}+C r_{i j}$, donde,

$R_{i j}$ es la respuesta empírica o puntuación numérica y

$\mathrm{C}_{0}, \mathrm{C}$ son constantes.

Este segundo supuesto establece que la respuesta empírica es función lineal de la respuesta interna. Es decir, que la Ley Psicomotora es lineal. 
c) Constancia de los valores subjetivos $\mathrm{S}_{\mathrm{Ai}}, \mathrm{S}_{\mathrm{Bj}}$.

Este supuesto establece que el valor subjetivo de cada estímulo es estable al combinarlo con otros.

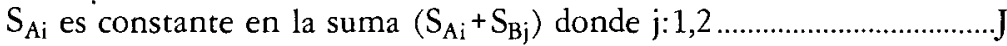

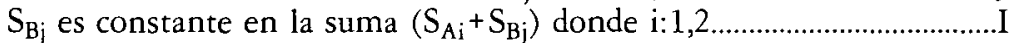

Partiendo de estos supuestos se enuncia el siguiente teorema.

Teorema I. - (Teorema del paralelismo)

Si $r_{i j}=S_{A i}+S_{B j}$ y $R_{i j}=C_{0}+C r_{i j}$ entonces

$\left(R_{i j}-R_{k j}\right)$ es constante para $j: 1,2$.

Este teorema nos asegura que fijando dos filas cualesquiera, la diferencia entre los elementos de estas filas es constante para toda columna j.

La demostración de este teorema es bastante simple.

Fijemos dos filas cualesquiera, i, k. Entonces

$\mathrm{R}_{\mathrm{ij}}=\mathrm{C}_{0}+\mathrm{C} \mathrm{r}_{\mathrm{ij}}=\mathrm{C}_{0}+\mathrm{C}\left(\mathrm{S}_{\mathrm{Ai}}+\mathrm{S}_{\mathrm{Bj}}\right)$

$\mathrm{R}_{\mathrm{kj}}=\mathrm{C}_{0}+\mathrm{C} \mathrm{r}_{\mathrm{kj}}=\mathrm{C}_{0}+\mathrm{C}\left(\mathrm{S}_{\mathrm{Ak}}+\mathrm{S}_{\mathrm{Bj}}\right)$

Restando miembro a miembro

$R_{\mathrm{ij}}-\mathrm{R}_{\mathrm{kj}}=\mathrm{C}\left(\mathrm{S}_{\mathrm{Ai}}-\mathrm{S}_{\mathrm{Ak}}\right)$

pero $S_{A i}$ es constante en la fila i y

$S_{A k}$ es constante en la fila $k$

luego, $R_{i j}-R_{k j}=C\left(S_{A i}-S_{A k}\right)$ es constante $V_{j}$

Como consecuencia de este teorema las gráficas de los datos en un diseño factorial deberán ser curvas paralelas.

El mismo resultado se obtiene si, en lugar de fijar dos filas, fijamos dos columnas. Es decir el Teorema I puede ser enunciado también así;

Si $r_{i j}=S_{A i}+S_{B j}$ y $R_{i j}=C_{0}+C r_{i j}$ entonces

$R_{i j}-R_{i p}$ es constante para $i: 1,2$

El paralelismo de las filas y de las columnas en un diseño factorial puede ser analizado mediante un análisis de varianza. Si los datos verifican este paralelismo la interacción debe ser no significativa.

La importancia del Teorema I estriba en que el modelo aditivo de integración puede ser puesto a prueba. Si en un diseño factorial se observa paralelismo, es decir, el valor F de la interacción es no significativo, se apoyan los supuestos del modelo. Por el contrario, si no existe paralelismo tendremos que rechazar el modelo.

Como puede observarse, en el análisis precedente no se hay utilizado valores numéricos como medida de los estímulos. Tanto A como B, en sus distintas categorías, pueden ser palabras, fras̀es, dibujos, etc. El análisis realizado recae exclusivamente sobre las respuestas empíricas o puntuaciones $\mathrm{R}_{\mathrm{ij}}$. En esta aproximación funcional de la medida no han una asignación numérica directa a los distintos estímulos, sino que la medida de los mismos se deriva del modelo mismo. La escala de medida para los estímulos nos la proporciona el siguiente teorema.

\section{Teorema II}

Si $r_{i j}=S_{A i}+S_{B j}$ y $R_{i j}=C_{0}+C r_{i j} \quad$ entonces

$\mathrm{R}_{\mathrm{j}}=\mathrm{C}_{0}+\mathrm{C} \mathrm{S}_{\mathrm{Bj}}$, donde

$\mathrm{C}_{0}^{\mathrm{f}}, \mathrm{C}$ son constantes y $\mathrm{R}_{\mathrm{j}}$ es la media de los elementos de la columna j.

La demostración de este teorema es bastante simple

$$
\begin{aligned}
& \mathrm{R}_{\mathrm{i}}=\left(\sum_{\mathrm{i}=}^{\mathrm{I}} \mathrm{R}_{\mathrm{ij}}\right) / \mathrm{I}=1 / \mathrm{I} \sum_{\mathrm{i}}^{\mathrm{I}}\left[\mathrm{C}_{0}+\mathrm{C}\left(\mathrm{S}_{\mathrm{Ai}}+\mathrm{S}_{\mathrm{Bj}}\right)\right]= \\
& \mathrm{C}_{0}+\mathrm{C} \sum \mathrm{S}_{\mathrm{Ai}} / \mathrm{I}+\mathrm{C} \mathrm{\textrm {S } _ { \mathrm { Bj } }}=\mathrm{C}_{0}+C \mathrm{~S}_{\mathrm{A}}+\mathrm{C} \mathrm{S}_{\mathrm{Bi}}
\end{aligned}
$$


Para $\mathrm{j}: 1,2$ J la suma $\left(\mathrm{C}_{0}+\mathrm{C} \mathrm{S}_{\mathrm{A}^{+}}\right)$es constante.

Luego $R_{\mathrm{j}}=\mathrm{C}_{0}^{i}+C S_{\mathrm{Bj}}$

Este teorema nos asegura que, si se mantienen los supuestos del modelo, las medias de las columnas son función lineal de los valores subjetivos de los estímulos situados en las columnas, lo cual significa que estas medias marginales son una escala de intervalos de los valores subjetivos $S_{B}$.

El mismo teorema puede aplicarse a las filas obteniendo una escala de intervalos para los valores subjetivos $S_{A}$.

\section{El Modelo Multiplicativo}

Al igual que en el modelo aditivo, consideramos dos continuos, $A$ y $B$, y una respuesta empírica, $R_{i j}$, dada a un par de estímulos, $A_{i}, B_{j}$.

Los supuestos del modelo son:

a) $r_{i j}=S_{A i} \cdot S_{B j}$

b) $\mathrm{R}_{\mathrm{ij}}=\mathrm{C}_{0}+\mathrm{C} \mathrm{r}_{\mathrm{ij}}$

c) Estabilidad o constancia de los valores subjetivos, $\mathrm{S}_{\mathrm{Ai}}, \mathrm{S}_{\mathrm{Bi}}$.

\section{Teorema III}

Si $r_{i j}=S_{A i} \cdot S_{B j}$ y $R_{i j}=C_{0}+C r_{i j}$ entonces $R_{i j}=C_{0}+C S_{A i} S_{B j}$

Esto significa que todas las puntuaciones de una misma fila, i, son función lineal de los valores subjetivos $S_{B}$, ya que el valor subjetivo $S_{A i}$ es constante en la fila $\mathrm{i}$.

La gráfica de un diseño factorial será entonces un haz de rectas con la misma ordenada en el origen, $\mathrm{C}_{0}$, y distinta pendiente, $\mathrm{CS}_{\mathrm{Ai}}$.

No olvidemos que $S_{A i}$ es constante dentro de una misma fila pero cambia para i: 1,2

Igualmente que en el modelo aditivo, si los supuestos son ciertos, entonces las medias de las columnas, $\mathrm{R}_{\mathrm{j}}$, son una estimación de los valores subjetivos, $\mathrm{S}_{\mathrm{B} j}$, de los estímulos situados en las columnas, ya que

$$
R_{. j}=C_{0}+C^{\prime} S_{B j}
$$

Es decir, al igual que acontecía en el modelo aditivo, las medias de las columnas son función lineal de los valores subjetivos, $\mathrm{S}_{\mathrm{B} \text {; }}$, de los estímulos situados en las columnas.

Lo mismo puede decirse con respecto a las medias de los elementos de las filas.

Esto significa que las medias marginales son una escala de intervalos de los estímulos integrados:

Para terminar la exposición de la Teoría de la Integración de la Información, señalaremos que una de las grandes ventajas que ofrece esta teoría es su gran campo de aplicación, ya que los estímulos integrados pueden ser de cualquier tipo. Anderson y sus colaboradores han desarrollado un amplio programa de investigaciones que abarca casi todos los campos de la Psicología: Psicolingüística (Anderson 1962; Oden \& Anderson, 1974), formación de actitudes (Anderson 1973), psicología evolutiva (Anderson \& Butzin, 1978; Anderson \& Cuneo, 1978), psicofísica (Anderson 1970, 1975, 1976) y otras muchas áreas de la Psicología que no vamos a mencionar por no alargar demasiado esta exposición.

Pasamos ahora a describir lo que constituye el objetivo fundamental de este trabajo. 


\section{COMPARACION DE LAS ESCALAS DE MEDIDA OBTENIDAS POR EL METODO DE THURSTONE Y DE ANDERSON, RESPECTIVAMENTE}

\section{Objetivo}

El objetivo de esta investigación es comprobar si dos escalas de medida ofrecen la misma medición cuando se utilizan los mismos estímulos y los mismos sujetos. En los capítulos anteriores hemos presentado dos modelos que, desde supuestos distintos, permiten establecer una escala de intervalos en la medición de estímulos subjetivos. Si el continuo subjetivo puede ser medido debe, entonces, esperarse que exista una convergencia entre ambas escalas de medida. Es decir, debemos esperar que, tanto la Ley del Juicio Comparativo, como la Teoría de la Integración de la Información ofrezcan la misma escala de medida.

\section{Hipótesis}

Si la medición, $f(A)$, establecida para un continuo subjetivo, $A$, mediante la Ley del Juicio Comparativo es una escala de intervalos, y si la medición, $g(A)$, establecida para el mismo continuo subjetivo, $A$, mediante la Teoría de la Integración de la Información es una escala de intervalos, entonces debe existir una función, $h$,

$$
h: f(A) \quad g(A)
$$

tal que h sea una transformación admisible para la escala de intervalos. Es decir,

$$
\mathrm{h}(\mathrm{x})=\alpha \mathrm{x}+\beta, \alpha>0
$$

Esta hipótesis lleva implícita la utilización del mismo grupo de sujetos. para obtener $f(A)$ y $g(A)$.

La misma hipótesis se establece para otro continuo subjetivo, B.

\section{Muestra}

La muestra está formada por 60 alumnos de primer curso de la Facultad de Psicología (U. Complutense), de los cuales 49 son mujeres y 11 son varones. La edad oscila entre 17 y 39 años, aunque el 83 por 100 del grupo está entre 18 y 20 años.

\section{Estímulos}

Se han utilizado dos continuos, A y B. El continuo A ha sido llamado «Zona Política» y consta de cuatro categorías. El continuo $\mathrm{B}$ ha sido llamado «Adjetivo» y consta de tres categorías.

A: Derecha (D), Centro Derecha (C.D), Centro Izquierda (C.I), Izquierda (I)
B: Honesto (H), Eficaz (E), Inteligente (Int)

Conviene aclarar que la utilización de dos continuos, A y B, responde a un requerimiento de la Teoría de la Integración de la Información. 
Así pues, $\mathrm{f}(\mathrm{A})$ y $\mathrm{f}(\mathrm{B})$ șerán las escalas de medida obtenidas para $\mathrm{A}$ y $B$, respectivamente, utilizando la Ley del Juicio Comparativo, y $g(A)$ y $g(B)$ serán las escalas obtenidas para $A$ y $B$, respectivamente, utilizando la Teoría de la Integración de la Información.

5. Obtención de las escalas de medida, $f(A)$ y $f(B)$, bajo los supuestos del Caso V de Thurstone.

\subsection{Obtención de $f(A)$}

El continuo A (Zona Política) consta de cuatro estímulos o categorías, por tanto tendremos $\mathrm{C}_{4,2}=6$ pares distintos de estímulos. Estos pares son:

Derecha, Centro Derecha

Derecha, Centro Izquierda

Derecha, Izquierda

Centro Derecha, Centro Izquierda

Centro Derecha, Izquierda

Centro Izquierda, Izquierda

Los seis pares fueron presentados a los sesenta sujetos. Cada sujeto recibía una lista en la cual, tanto el orden de los pares como el de los elementos dentro de cada par, fue aleatorio. Es decir, todos los sujetos recibían los mismos pares pero presentados de distinta forma.

A cada sujeto se le pidió que subrayase dentro de cada par aquella zona o espacio político que prefiriese al comparar los dos elementos que componían cada par. Se les dijo explícitamente que debían subrayar un solo elemento en cada par y que no debía quedar ningún par sin subrayar.

Con los datos obtenidos se construyeron las matrices F, P y $Z$.

La matriz $\mathrm{F}$ ofrece la distribución de frecuencias obtenida. El elemento $\mathrm{f}_{\mathrm{ij}}$ representa el número de veces que el estímulo i fue preferido al estímulo j, donde, siguiendo la notación matricial habitual, i representa la fila y j representa la columna.

La matriz $P$ es tal que el elemento $\mathrm{p}_{\mathrm{ij}}=\mathrm{f}_{\mathrm{ij}} / \mathrm{N}$ donde $\mathrm{N}$ es el número de sujetos.

La matriz $Z$ es tal que el elemento $z_{i j}$ es la puntuación típica que deja debajo de sí una proporción igual a $\mathrm{p}_{\mathrm{ij}}$, bajo el supuesto de normalidad.

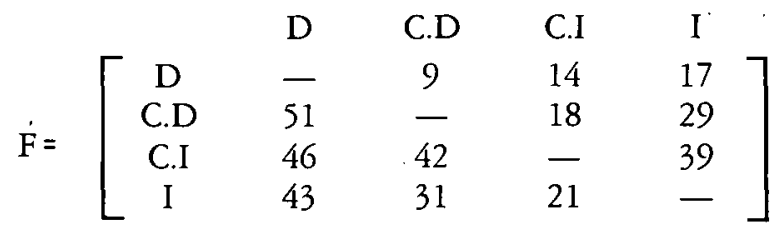

$$
\begin{aligned}
& \mathrm{P}=\left[\begin{array}{ccccc} 
& \mathrm{D} & \mathrm{C} . \mathrm{D} & \mathrm{C} . \mathrm{I} & \mathrm{I} \\
\mathrm{D} & - & .150 & .233 & .283 \\
\mathrm{C} . \mathrm{D} & .850 & - & .300 & .483 \\
\mathrm{C} . \mathrm{I} & .767 & .700 & - & .650 \\
\mathrm{I} & .717 & .517 & .350 & -
\end{array}\right] \\
& \mathrm{Z}=\left[\begin{array}{ccccc} 
& \mathrm{D} & \mathrm{C} . \mathrm{D} & \mathrm{C} . \mathrm{I} & \mathrm{I} \\
\mathrm{D} & - & -1.04 & -.73 & -.57 \\
\mathrm{C} . \mathrm{D} & 1.04 & - & -.52 & -.04 \\
\mathrm{C} . \mathrm{I} & .73 & .52 & - & .39 \\
\mathrm{I} & .57 & .04 & -.39 & -
\end{array}\right]
\end{aligned}
$$


Como puede observarse; en las matrices F, P y Z los elementos de la diagonal principal no aparecen. Ello es así porque no se compara un estímulo consigo mismo.

Sea $S_{i}={ }_{j} z_{i j} / 4$ la media de los elementos de la matriz $Z$ que están situados en la fila $\mathrm{i}$. Bajo los supuestos del Caso $\mathrm{V}$ de Thurstone, $\mathrm{S}_{\mathrm{i}}$ es una medida del estímulo subjetivo situado en la fila i (Véase Torgerson 1958, Yela 19).

En nuestro caso

$\mathrm{S}_{\mathrm{i}}=-.585, \mathrm{~S}_{2}=.12, \mathrm{~S}_{3}=.41, \mathrm{~S}_{4}=.055$

Situando el cero en el valor más bajo de la escala tendremos

$f\left(A_{i}\right)=S_{i}+0.585$

Ordenando de menor a mayor, la escala obtenida es
$A:^{*}$
Derecha
Izquierda
0.640
C. Derecha
C. Izquierda
$f(A)$ :
0
0.705
0.995

Estos valores constituyen una escala de intervalos del continuo subjetivo A (Zona Política). Ahora bien, estos valores, así obtenidos, se apoyan en unos supuestos, los del Caso V de Thurstone. En la medida en que se mantengan estos supuestos la escala obtenida tendrá un valor. Por esta razón conviene hacer el ajuste de los datos empíricos a los teóricos, esto es, aquellos bajo los*supuestos del Caso $\mathrm{V}$ de Thurstone.

En nuestro caso hemos obtenido

$$
\mathrm{X}^{2}=7.94 \quad \mathrm{~g} \cdot 1=3 \quad \mathrm{P}<.05 .
$$

$\mathrm{Si} \mathrm{X}^{2}$ es significativo quiere decir que existe discrepancia entre los datos empíricos y los teóricos. Es decir, no hay un buen ajuste de nuestros datos a aquellos que se debían obtener según los supuestos en que nos hemos apoyado, y, por tanto, nuestra escala no tiene el soporte teórico que hemos asumido.

Puesto que nuestro objetivo es comparar esta escala con la obtenida bajo los supuestos de la Teoría de la Integración de la Información, volveremos sobre este punto en la discusión de los resultados, una vez realizada la comparación de ambas escalas.

Veamos ahora cómo obtenemos los valores $\mathrm{f}(\mathrm{B})$ para el estímulo $\mathrm{B}$.

\subsection{Obtención de $f(B)$}

Puesto que el continuo B (Adjetivo) consta de tres categorías o estímulos tenemos $C_{3,2}=3$ pares distintos de estímulos. Estos pares son:

Honesto, Eficaz

Honesto, Inteligente

Eficaz, Inteligente

Al igual que con el continuo $\mathrm{A}$, los pares fueron dispuestos aleatoriamente, de modo que cada sujeto recibía los mismos pares pero presentados de forma diferente, tanto en lo referente al orden de los pares como al de los elementos dentro de cada par.

Cada sujeto debía subrayar un adjetivo dentro de cada par, aquel que prefiriese, teniendo en cuenta que estos adjetivos se referían o caracterizaban a un hipotético político. Expresamente se dijo que considerasen que el político era hipotético y por tanto podía situarse en cualquier espacio político. Igualmente se les dijo que debían subrayar un solo adjetivo dentro de cada par y que no podía quedar ningún par sin subrayar. 
Con los datos obtenidos se construyen las matrices F, P y Z, cuyo significado es el mismo que para el caso del continuo $\mathrm{A}$.

$$
\begin{aligned}
& \mathrm{F}=\left[\begin{array}{cccc}
\mathrm{H} & \mathrm{H} & \mathrm{E} & \text { Int } \\
\mathrm{E} & 25 & 35 & 33 \\
\text { Int. } & 27 & 26 & 34
\end{array}\right] \\
& \mathrm{P}=\left[\begin{array}{cccc}
\mathrm{H} & - & .583 & .550 \\
\mathrm{E} \cdot & .417 & - & .567 \\
\text { Int. } & .450 & .433 & -
\end{array}\right] \\
& \mathrm{Z}=\left[\begin{array}{cccc}
\mathrm{H} & \mathrm{E} & \text { Int. } \\
& & & \\
\mathrm{H} & -.21 & -21 & .13 \\
\text { Int. } & -13 & -.17 & -
\end{array}\right]
\end{aligned}
$$

Sea $\mathrm{S}_{\mathrm{i}}=; \mathrm{z}_{\mathrm{ij}} / 3$ la media de los elementos de la matriz $\mathrm{Z}$ que están situados en la fila i. Como ya se dijo, $S_{\mathrm{i}}$ es una medida del estímulo situado en la fila $\mathrm{i}$.

En nuestro caso

$\mathrm{S}_{\mathrm{i}}=0.113, \mathrm{~S}_{2}=-0.013, \mathrm{~S}_{3}=-0.1$

Situando el cero en el valor más bajo de la escala tendremos $f\left(B_{i}\right)=S_{i}+0.1$

La escala obtenida, ordenada de menor a mayor, es

$$
\begin{array}{rccc}
\text { B: } & \text { Inteligente } & \text { Eficaz } & \text { Honesto } \\
\mathrm{f}(\mathrm{B}): & 0 & 0.087 & 0.213
\end{array}
$$

$\mathrm{Al}$ igual que con el continuo $\mathrm{A}$, hemos realizado el ajuste de los datos empíricos a los teóricos bajo los supuestos del Caso V. En este caso hemos obtenido

$$
\mathrm{X}^{2}=3.84 \quad \mathrm{~g} .1=1 \quad \mathrm{p}>.05
$$

$\mathrm{X}^{2}$ no es significativo, lo cual quiere decir que existe un buen ajuste entre nuestros datos y aquellos que se deben obtener según los supuestos en que nos hemos apoyado y, por tanto, nuestra escala tiene el soporte teórico que hemos asumido.

6. Obtención de las escalas de medida, $\mathrm{g}(\mathrm{A})$ y $\mathrm{g}(\mathrm{B})$, bajo los supuestos de la Teoría de la Integración de la Información

Para obtener las escalas $g(A)$ y $g(B)$ construimos una lista con todos los posibles parés $A_{i}, B_{j}$, donde i:1, 2, 3, 4 y j:1, 2, 3 . 
Por tanto los pares posibles son 12. Estos pares son:

Derecha, Honesto

Derecha, Eficaz

Derecha, Inteligente

C. Derecha, Honesto

C. Derecha, Eficaz

C. Derecha, Inteligente

C. Izquierda, Honesto

C. Izquierda, Eficaz

C. Izquierda, Inteligente

Izquierda, Honesto

Izquierda, Eficaz

Izquierda, Inteligente

Los pares fueron dispuestos aleatoriamente, de modo que todos los sujetos recibieron la misma lista pero en forma diferente. Para unos sujetos los pares tenían como primer elemento el adjetivo y para otros el primer elemento era el espacio político.

La tarea de cada sujeto consistió en puntuar desde 0 hasta 10 a un hipotético político que estuviese caracterizado por los dos elementos de que constaba el par. Así pues, cada sujeto otorgó doce puntuaciones, una por cada par. En la Tabla 1 se presentan las medias de las puntuaciones otorgadas por los sesenta sujetos a cada par.

TABLA 1

\begin{tabular}{|c|c|c|c|c|c|}
\hline & $\mathrm{D}$ & C.D & C.I & I & B \\
\hline $\mathrm{H}$ & 5.667 & 6.150 & 6.850 & 7.116 & 6.446 \\
\hline E & 5.233 & 5.856 & 6.666 & 6.866 & 6.155 \\
\hline 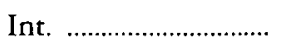 & 4.867 & 5.750 & 6.530 & 6.633 & 5.945 \\
\hline A & 5.255 & 5.918 & 6.682 & 6.871 & \\
\hline
\end{tabular}

La fila A representa las medias de cada una de las categorías del continuo A (Zona Política). Estas medias constituyen según la Teoría de la Integración de la Información una escala de intervalos para el continuo «Zona Política».

La Columna B representa las medias de cada una de las categorías del continuo B (Adjetivo), y son, igualmente una escala de intervalos para este continuo.

Ahora bien, las medias marginales son una escala de intervalos para los estímulos situados en las filas o en las columnas bajo los supuestos del modelo aditivo o multiplicativo de integración de la información. Conviene, pues, poner a prueba nuestros datos para ver si se ajustan a alguno de los dos modelos.

La Gráfica 1 representa las medias de las casillas de la Tabla 1.

Como puede apreciarse, las líneas que unen las medias de las filas son aproximadamente paralelas, dentro de las fluctuaciones que se pueden esperar por azar. El paralelismo de los datos apoya (Teorema I) la hipótesis de un modelo aditivo de integración de la información. 


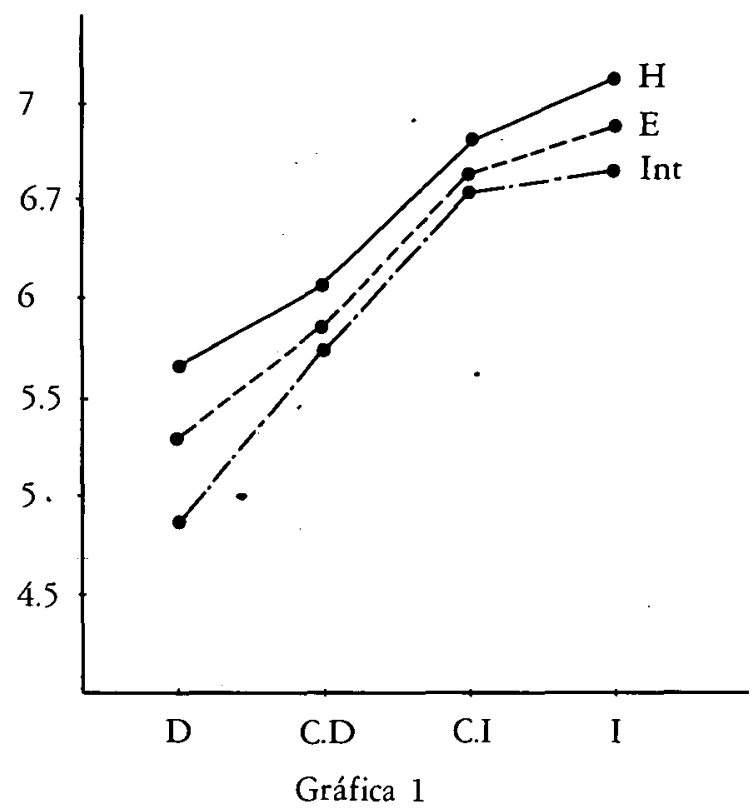

Bajo los supuestos del modelo aditivo podemos afirmar (Teorema II) que las medias de las filas y de las çolumnas son, respectivamente, una escala de intervalos para el continuo $\mathrm{B}$ y $\mathrm{A}$.

Ordenando de menor a mayor y situando el cero en el valor más bajo de la escala tenemos:

$g(A)=A_{i}-5.255$

$\mathrm{g}(\mathrm{B})=\mathrm{B}_{\mathrm{j}}-5.945$

Las escalas obtenidas son:
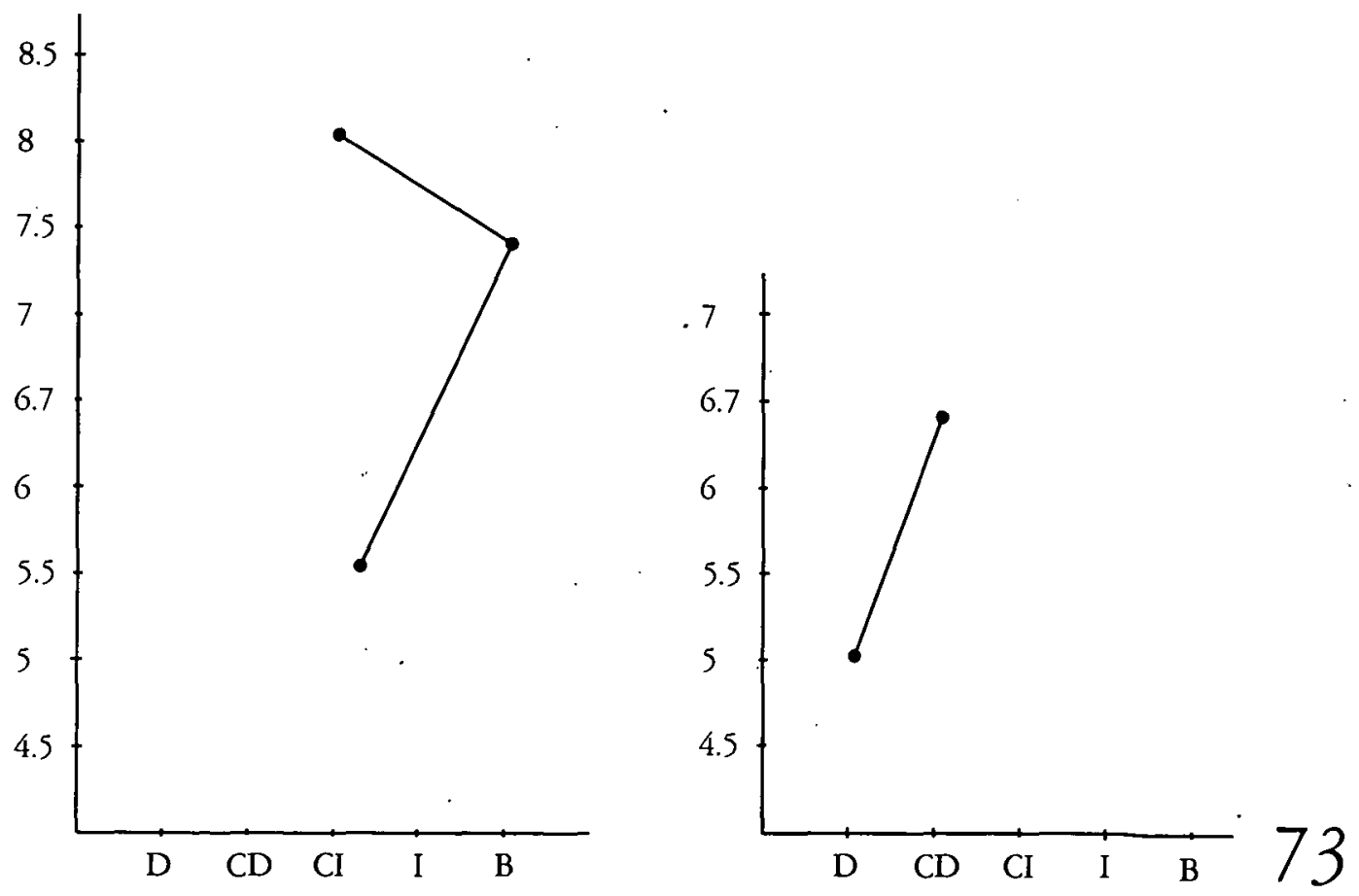


$\begin{array}{rcccc}\text { A: } & \text { Derecha } & \text { C. Derecha } & \text { C. Izquierda } & \text { Izquierda } \\ \mathrm{g}(\mathrm{A}): & 0 & 0.663 & 1.427 & 1.616\end{array}$

$\begin{array}{rccc}\text { B: } & \text { Inteligente } & \text { Eficaz } & \text { Honesto } \\ \mathrm{g}(\mathrm{B}): & 0 & 0.210 & 0.501\end{array}$

\section{Comparación entre las escalas de medida, f y $\mathrm{g}$}

Puesto que $f(A)$ y $g(A)$ son valores en escala de intervalos para unos mismos estímulos, entonces debe existir una transformación, h, admisible para dicha escala entre ambos conjuntos numréricos

$$
h: \mathrm{f}(\mathrm{A}) \mathrm{g}(\mathrm{A}) \quad \mathrm{y} \quad \mathrm{h}(\mathrm{x})=\alpha \mathrm{x}+\beta \quad, \quad \alpha>0
$$

e, igualmente, podemos decir con respecto a $f(B)$ y $g(B)$.

Para obtener ambas funciones se ha realizado un ajuste lineal mediante mínimos cuadrados.

Las gráficas 2 y 3 muestran, respectivamente, el diagrama de dispersión de los puntos $\left[f\left(A_{i}\right), g\left(A_{i}\right)\right]$ y $\left[f\left(B_{j}\right), g\left(B_{j}\right)\right]$

Las correlaciones y las rectas de regresión obtenidas han sido

$r_{A}=0.806$

$r_{B}=0.9999$

$$
\begin{aligned}
& \mathrm{g}^{\prime}\left(\mathrm{A}_{\mathrm{i}}\right)=1.428 \mathrm{f}\left(\mathrm{A}_{\mathrm{i}}\right)+0.091 \\
& \mathrm{~g}^{\prime}\left(\mathrm{B}_{\mathrm{j}}\right)=2.356 \mathrm{f}\left(\mathrm{B}_{\mathrm{j}}\right)+0.001
\end{aligned}
$$

\section{Discusión de los resultados}

Como puede observarse, para el continuo B la correlación obtenida, $r_{B}=0.999$, es casi perfecta. En el diagrama de dispersión vemos que los puntos están prácticamente sobre la recta $\mathrm{h}(\mathrm{x})=2.356 \mathrm{x}+0.001$, lo cual nos indica que, tanto la Teoría de la Integración de la Información como la Ley del Juicio Comparativo, nos han proporcionado, partiendo desde supuestos distintos, la misma escala de medida.

En el caso del continuo A no existe tal convergencia entre las dos escalas. La correlación obtenida, $\mathrm{r}_{\mathrm{A}}=0.806$, a pesar de ser alta, no lo es en la medida en que debiera serlo si ambas escalas fuesen la misma. El error que cometemos al pasar desde $f(A)$ a $g(A)$ con la recta $u(x)=1.428 x+0.091$ es aproximadamente un 35 por 100 de la varianza de $g(A)$, lo cual, desde nuestro punto de vista, es bastante.

Si observamos ambas escalas, $f(A)$ y. $g(A)$, vemos que ni siquiera son la misma escala considerándolas ordinalmente. El valor numérico asignado al estímulo «Izquierda» es el más alto en la escala $g$, mientras que es el tercero en la escala $f$, lo cual provoca varias inversiones entre los órdenes. De hecho, si transformamos los valores $f(A)$ y $g(A)$ en órdenes obtenemos una correlación ordinal $r_{s}=0.4$. Este resultado puede sorprender a primera vista puesto que considerando los datos en escala de intervalos hemos obtenido $r_{A}=0.806$, pero hay que tener en cuenta que la correlación $r_{A}=0.806$ es tan alta porque el punto $(0,0)$ es muy extremo y, por tanto, dirige los datos hacia la linealidad.

El mal ajuste entre $f(A)$ y $g(A)$, por otra parte, no debe sorprendernos, dado que, según vimos al obtener la escala $f(A)$, no existía el soporte teórico que habíamos supuesto. Si recordamos, el valor obtenido para $\mathrm{X}^{2}$ fue significativo lo cual indicaba que nuestros datos empíricos no se ajustaban a los datos teóricos bajo los supuestos del Caso $\mathrm{V}$ de Thurstone. 
Como conclusión final debemos decir que los resultados obtenidos en esta investigación son esperanzadores en cuanto que ofrecen un amplio apoyo a la posibilidad de medir estímulos subjetivos. Dos teorías, la Ley del Juicio Comparativo y la Teoría de la Integración de la Información, nos han proporcionado la misma escala de intervalos partiendo desde supuestos distintos, en el caso en que los datos empíricos han verificado ambos supuestos. Por el contrario, han ofrecido dos escalas de medida distintas en el caso en que los datos empíricos no han verificado los supuestos de àlguna de las dos teorías.

En este trabajo el diseño factorial utilizado, $3 \times 4$, es aproximado a los utilizados por Anderson, en lo que se refiere al numero de categorías que componen cada estímulo. La utilización de pocas categorías tiene una dificultad que no se nos puede pasar por alto, y es que una correlación con pocos puntos tiende a ser muy alta. En este sentido, las conclusiones aquí expuestas deben ser aceptadas con cierta cautela hasta que nuevos estudios, ampliando el número de categorías de los estímulos integrados, sean llevados a cabo.

\section{Referencias}

ANDERSON, N. H. «Application of an additive model to impression formation». Science, 1962, 138, 817-818.

- «Functional measurement and psychophysical judgment». Psychological Review, 1970, 77, 153-170.

- "Information integration theory applied to attitudes about U.S. presidents» J. of Educational Psychology, $1973,64,1-8$.

- "Algebraic models in perception», en E. C. Carterette y M. P. Friedman (eds.) Handbook of perception (Vol. 2), Nueva York, Academic Press, 1974 a.

- «Cognitive algebra», en L. Berkowitz (ed.): Advances in experimental social psychology, Vol. 7, Nueva York, Academic Press, 1974b.

- «Information integration theory: A brief survey», en D. Krantz, R. C. Atkinson, R. D. Luce y P. Suppes (eds.): Contemporary developmentsin matbematical psychology, Vol. 2. San Francisco: Freeman, 1974c.

- «On the role of context effects in psychophysical judgment». Psycbological Review, 1975, 82, 462-482.

- «Integration theory, functional measurement an the psychophysical law», en $H$. G. Geissler y Yu. M. Zabrodin (eds.): Advances in psychopbysics. Berlín: VEB Deutscher Verlag, 1976.

- Foundations of information integration theory. Nueva York: Academic Press, 1981.

- Methods of information integration theory. Nueva York: Academic Press, 1982.

ANDERSON, N. H. y BUTZIN, C. A. «Integration theory applièd to children's judgments of equity». Developmental Psychology, 1978, 14, 593-606.

ANDERSON, N. H. y CUNEO, D. O. "The Height+Width rule in children's judgments of quantity». J. of Experimental Psychology: General, 1975, 107, 335-378.

Oden, G. C., y ANDERSON, N. H. «Integration of semantic constraints». J. of Verbal Learning and Verbal Bebavior, 1974, 13, 138-148.

Turstone, L. L. «A law of comparative judgment». Psychological Review, 1927a, 34, 273-286

- «Psychophysical Analysis». Amer. J. Psychology, 1927b, 38, 368-389.

- «Equally often noticed differences». J. of Educational Psychology, 1927c, 18, 289-293.

- The measurement of values. University of Chicago Press: Chicago y Londres, 1959.

Torgerson, W. S. Theory and Metbods of Scaling. Nueva York, Wiley, 1958.

Yela, M. «La ley de los juicios comparativos y la construcción de escalas psicológicas». Revista de Psicología General y Aplicada, vol. 21, 1966, 84, 659-690. 